Article

\title{
Fermentation of Plant Extracts Supplemented with Milk Components by Lactic Acid Bacteria Produces Soluble Agonists for Toll-like Receptor 2 Possibly Suitable for Cosmetics
}

\author{
Kanako Matsunaga and Yasuhiko Komatsu *(D)
}

Development Research Department, Snowden Co., Ltd., 3-7-16 Iwamoto-cho, Chiyoda-ku, Tokyo 101-0032, Japan; k_matsunaga@snowden.co.jp

* Correspondence: y_komatsu@snowden.co.jp; Tel.: +81-3-3866-2828

check for updates

Citation: Matsunaga, K.; Komatsu, Y. Fermentation of Plant Extracts Supplemented with Milk Components by Lactic Acid Bacteria Produces Soluble Agonists for Toll-like Receptor 2 Possibly Suitable for Cosmetics. Fermentation 2022, 8, 111. https://doi.org/10.3390/ fermentation 8030111

Academic Editor: Plessas Stavros

Received: 30 December 2021

Accepted: 4 March 2022

Published: 5 March 2022

Publisher's Note: MDPI stays neutral with regard to jurisdictional claims in published maps and institutional affiliations.

Copyright: (c) 2022 by the authors. Licensee MDPI, Basel, Switzerland. This article is an open access article distributed under the terms and conditions of the Creative Commons Attribution (CC BY) license (https:// creativecommons.org/licenses/by/ $4.0 /)$.

\begin{abstract}
Stimulation of Toll-like receptor 2 (TLR2) on epidermal keratinocytes results in the tightening of cell-cell junctions between keratinocytes; therefore, appropriate agonists for TLR2 could be promising ingredients for cosmetics. However, a method to produce significant amounts of soluble TLR2 agonists using materials that are suitable for preparing cosmetics has not yet been developed. In this study, we tried to identify appropriate lactic acid bacterial strains and media for fermentation to obtain soluble TLR2 agonists from traditional fermented foods and natural food sources. We found that Lactobacillus delbrueckii subsp. lactis TL24 (TL24) and a combination of hot water extracts of asparagus edible stem and cow skimmed milk were the best strain and culture medium, respectively, for this purpose. The TL24 ferments effectively stimulated TLR2 in HEK293 reporter cells expressing human TLR2 on their surface and also inhibited paracellular molecular transfer in a cell sheet of human primary keratinocytes. Since these effects of the TL24 ferments were suppressed by antiTLR2 neutralizing antibodies, it is proposed that TL24 ferments elicit these effects via TLR2. Taken together, these results suggest that TL24 ferments containing soluble TLR2 agonists are potential ingredients for cosmetics.
\end{abstract}

Keywords: lactic acid bacteria; epidermal keratinocyte; fermentation; tight junction; toll-like receptor 2

\section{Introduction}

An important requirement for skincare cosmetics is the maintenance of the skin barrier. The skin barrier protects the human body from attacks by the external environment (e.g., pathogens, physical traumas, and ultraviolet radiation) [1,2], prevents water transpiration from the surface of the skin, and retains moisture [2]. To moisten the skin, it is important to use different types of moisturizers in appropriate combinations, such as emollients, humectants, and occlusives [2,3]. In addition to this physical approach, it is important to maintain the barrier ability of the skin itself.

The skin is the largest organ of the human body, and its outermost layer is the epidermis, consisting of a highly stratified epithelium [4]. The basal layer of the skin epidermis (stratum basale), which is attached to the basement membrane, consists of mitotically active stem cells that produce undifferentiated keratinocytes. Keratinocytes are mitotically active and produce cells that sequentially differentiate into three histologically different outer layers-the innermost spinous layer (stratum spinosum), the granulosum layer (stratum granulosum), and finally the horny layer [stratum corneum (SC)]—by a finely tuned process [4]. The physiological skin barrier is mainly attributable to the SC and stratum granulosum [5]. SC consists of enucleated corneocytes in a lipid matrix. Keratinocytes in the granulosum form tight junctions (TJs) between cells, which form a strong barrier for the paracellular transfer of molecules [5]. Disruption of such structures is harmful for the skin; therefore, substances that can support the integrity of the skin are very useful as ingredients in cosmetics. Since TJ consists of several structural proteins, including claudins [6,7], 
occludin [8], and ZO-1 [9], which forms protein complexes tethering adjacent cells, agents upregulating these proteins might be candidates for such ingredients.

In the granulosum layer, TJs between keratinocytes have been reported to be tightened by the stimulation of their toll-like receptor 2 (TLR2) [10,11]. TLR2 is a cell surface receptor that mainly recognizes microbes, including Gram-positive bacteria, and plays an important role in innate immunity [12]. Although the main cell types expressing TLR2 are immune cells such as macrophages, monocytes, dendritic cells, and neutrophils [9], TLR2 is also expressed in epidermal keratinocytes [13], intestinal epidermal cells [14], and pulmonary alveolar epithelial cells [15]. When TLR2 is stimulated in immune cells, the immune response is triggered, including phagocytosis of pathogens, cytokine secretion, and presentation of antigens to professional immune cells, which results in inflammation [12]. By contrast, stimulation of TLR2 in epithelia is not directly related to inflammation but tightens the cellular barrier, which is another aspect of the defense mechanism of the skin [10,11].

Yuki et al. [10] and Kuo et al. [11] reported that TLR2 agonists are responsible for the rapid and chronic tightening of TJs in skin keratinocytes, suggesting that TLR2 agonists without pathogenic properties might be useful for skincare purposes by stimulating the skin's self-defense mechanism. Moreover, Kuo et al. [11] demonstrated that the stimulation of TLR2 plays a role in the wound repair response in atopic dermatitis (AD) patients. Therefore, appropriate TLR2 agonists are thought to be beneficial not only as cosmetics but also as AD therapeutics. However, in their studies, either peptidoglycan prepared from pathogenic Staphylococcus aureus or the synthetic ligand Pam3CSK4 was used as a TLR2 agonist. Moreover, to the best of our knowledge, no compounds with TLR2stimulating activity suitable for manufacturing cosmetics have been reported to date. In the present study, we tried to produce soluble TLR2 agonists using natural raw materials that are suitable for use in cosmetics. As a result, we found that the fermentation of plant extracts supplemented with milk components by appropriate lactic acid bacteria could produce significant amounts of soluble TLR2 agonists. Since such ferments contain soluble TLR2 agonists that can strengthen the barrier function of the cellular sheet of keratinocytes, they might be novel ingredients of skincare cosmetics.

\section{Materials and Methods}

\subsection{Lactic Acid Bacteria}

The lactic acid bacteria used in this study were kindly provided by Prof. Taku Miyamoto at Kurashiki Sakuyo University (Okayama, Japan); they were isolated from a variety of traditional fermented foods worldwide, and their culture supernatants were examined using a TLR2-stimulating assay described below. Lactobacillus delbrueckii subsp. lactis TL24 (TL24), whose culture supernatant has strong TLR2-stimulating activity, was isolated from Georgian traditional Sulguni cheese. The species of this strain was identified by comparing the 16S ribosomal RNA and gyrase $B$ gene sequences of the strain with those of other sequenced bacteria available in the DNA sequence database [DDBJ/ENA(EMBL)/GenBank].

\subsection{Normal Human Epidermal Keratinocytes}

The normal human epidermal keratinocytes used in this study, NHEK (NB), were purchased from Kurabo (Osaka, Japan) and were cultured in DermaLife K keratinocyte medium (Lifeline Cell Technology, Frederick, MD, USA) at $37^{\circ} \mathrm{C}$ and $5 \% \mathrm{CO}_{2}$ in a humidified chamber. All experiments were performed with cells passaged six times or less.

\subsection{Plant Extracts and Milk Materials}

A variety of plant materials, namely, from asparagus (stem of edible part), broccoli (stem of edible part), cabbage (edible part), carrot (edible part, root), daikon radish (edible part, root), Chinese cabbage (edible part), apple (fruit), eggplant (fruit), cucumber (fruit), lemon balm (leaf), orange (fruit), cherry (leaf), tomato (fruit), and grape (fruit), were subjected to hot water extraction. Unless otherwise mentioned, the plant bodies were mixed with purified water at a weight ratio of $1: 2$ and autoclaved $\left(121^{\circ} \mathrm{C}\right.$ for $\left.15 \mathrm{~min}\right)$. 
After the temperature was lowered to $40{ }^{\circ} \mathrm{C}$ or lower, the plant extracts were squeezed through a polypropylene mesh. The extract was stored at $-20^{\circ} \mathrm{C}$ or lower until further use. Among these plants, we chose asparagus as the main target for extensive examination, because (1) asparagus extracts have been used in cosmetics as skin conditioning agents [16]; (2) since the bottom part of the spear of asparagus is normally trimmed and discarded before shipment, the usage of these parts meets the policy of sustainable development goal (SDG) 12, namely, "responsible consumption and production" [17].

Several milk powders, namely, cow skimmed milk powder (Takanashi Milk Products, Kanagawa, Japan), cow whole milk powder (Yotsuba Milk Products, Hokkaido, Japan), goat whole milk powder (Meyenberg, Turlock, CA, USA), camel whole milk powder (Aadvik Foods \& Products Pvt. Ltd., Rajasthan, India), and cow cheese whey powder (Yotsuba Milk Products), were used in this study. Sheep whole milk powder was produced by lyophilizing sheep whole milk purchased from Matsuyama Farm (Hokkaido, Japan). Yogurt whey powder was produced by lyophilizing whey liquid obtained from plain yogurt (Takanashi Milk Products). Oat milk powder (GODO Co., Ltd., Tokyo, Japan) was used as a milk-like plant-derived material.

\subsection{Bacterial Culture and Fermentation}

Lactic acid bacteria were cultured in De Man, Rogosa, and Sharpe (MRS) medium (Difco) at their preferred temperature $\left(30^{\circ} \mathrm{C}\right.$ or $37^{\circ} \mathrm{C}$, depending on the strains) under static conditions for $24 \mathrm{~h}$ and used as the seed culture (MRS seed). For fermentation under static conditions, an MRS seed equivalent to $2 \%$ of the fermentation volume was added to the plant extracts with or without milk components after sterilization $\left(110^{\circ} \mathrm{C} \times 20 \mathrm{~min}\right)$, and fermentation was performed at the bacteria preferred temperature $\left(30^{\circ} \mathrm{C}\right.$ or $\left.37^{\circ} \mathrm{C}\right)$ for $24 \mathrm{~h}$. For fermentation by TL24 using a 5 L jar fermenter (Bioneer-Neo, Marubishi Bioengineering, Tokyo, Japan), an MRS seed equivalent to $2 \%$ of the fermentation volume was added to a sterilized asparagus extract containing 3\% skimmed milk, and the mixture was incubated at $37^{\circ} \mathrm{C}$ for $24 \mathrm{~h}$ in static conditions to obtain an asparagus seed culture. After the vessel of the fermenter was filled with $2.5 \mathrm{~L}$ of asparagus extract and $3 \%$ skimmed milk, sterilized at $110^{\circ} \mathrm{C}$ for $20 \mathrm{~min}$, and cooled to $37^{\circ} \mathrm{C}, 50 \mathrm{~mL}$ of the asparagus seed culture was inoculated. Fermentation was performed under mild stirring conditions $(80 \mathrm{rpm})$ at $37^{\circ} \mathrm{C}$ for $24 \mathrm{~h}$.

\subsection{TLR2- and TLR4-Stimulating Activities}

TLR2-stimulating activity was determined using a reporter cell system, HEK Blue ${ }^{\text {TM }}$ hTLR2 cells (InvivoGen, Toulouse, France), according to the manufacturer's instructions. In brief, the cells were cultivated in Dulbecco's modified Eagle's medium (Sigma-Aldrich, St. Louis, MO, USA) supplemented with a mixture of selective antibiotics for keeping the persistent expression of transgenes (HEK Blue selection, InvivoGen), in addition to other antibiotics for preventing the growth of microorganisms (penicillin, Fujifilm-Wako, Osaka, Japan; streptomycin, Fujifilm-Wako; and normocin, InvivoGen), and 10\% fetal bovine serum (Equitech Bio, Kerrville, TX, USA) at $37^{\circ} \mathrm{C}$ in a $5 \% \mathrm{CO}_{2}$ atmosphere in a humidified chamber. For the assay, the cells were seeded in each well of a 96-well cell culture plate at a density of $2 \times 10^{5}$ cells $/ \mathrm{mL}$ in $100 \mu \mathrm{L}$ of the culture medium. After $2 \mathrm{~d}, 100 \mu \mathrm{L}$ of the test samples, sterilized by membrane filtration (pore size $=0.2 \mu \mathrm{m}$ ) and diluted in the culture medium, was added to each well. As positive and negative controls, $200 \mathrm{ng} / \mathrm{mL}$ of Pam3CSK4 (final concentration $100 \mathrm{ng} / \mathrm{mL}$ ) and the medium were used, respectively. After an additional $1 \mathrm{~d}$ of cultivation, $20 \mu \mathrm{L}$ of the conditioned medium from each well was transferred to a fresh 96-well assay plate, $180 \mu \mathrm{L}$ of Quanti Blue Solution (InvivoGen) was added, and the plate was incubated for $30 \mathrm{~min}$ at $37^{\circ} \mathrm{C}$. The absorbance of each well was determined at $655 \mathrm{~nm}$ using a microplate reader (Multiskan GO, Thermo Fisher Scientific, Waltham, MA, USA), and the average value of the negative control wells was subtracted from the values of all wells. TLR2-stimulating activity is expressed as the relative value to the average values of the positive control. When examining TLR4-stimulating activity, HEK Blue hTLR4 cells (InvivoGen) and 1000 ng/mL LPS (Lipopolysaccharide 
from Escherichia coli 0111:B4; Sigma-Aldrich) were used instead of HEK Blue hTLR2 and $100 \mathrm{ng} / \mathrm{mL}$ Pam3CSK4, respectively.

\subsection{Transcellular Molecular Transfer in Keratinocytes}

NHEK (NB) cells were seeded in a BioCoat Control Culture insert (0.4 $\mu \mathrm{m}$ PET membrane, 24 wells; Corning, NY, USA) and cultured for $4 \mathrm{~d}$ until they reached confluence at $37^{\circ} \mathrm{C}$ and $5 \% \mathrm{CO}_{2}$ in a humidified chamber. To examine the effect on transcellular molecular transfer, both the inner and the outer solutions were changed to solutions containing $1.8 \mathrm{mM} \mathrm{CaCl}_{2}$, which was used to stimulate the differentiation of keratinocytes, with or without diluted test samples. After $3 \mathrm{~d}$ of incubation, $1 / 50$ volume of $1 \%$ fluorescein sodium was added to the inner solution (final concentration $=0.02 \%$ ). After $30 \mathrm{~min}$ at $37^{\circ} \mathrm{C}$, the outer solution was collected and diluted 10-fold with PBS, and its fluorescence was determined using a real-time PCR apparatus (Thermal Cycler Dice Real-time System II; Takara Bio, Shiga, Japan). To evaluate whether TLR2-stimulating activity was the underlying mechanism, $1 \mu \mathrm{g} / \mathrm{mL}$ of neutralizing antibody against human TLR2 (Clone 383936; R\&D Systems, Minneapolis, MN, USA) was added to the samples.

\subsection{Statistics}

Statistical differences between the control group and each test group were analyzed using the Dunnett's test. If statistical comparisons between all groups were necessary, Scheffe's test was employed. All statistical analyses were performed using the KaleidaGraph software (Synergy Software, Ver4.5, Reading, PA, USA). Differences between groups were considered statistically significant when the significance probability was less than 0.05 .

\section{Results}

\subsection{Lactic Acid Bacteria Produce Soluble Factors with TLR2-Stimulating Activity}

Figure 1A shows the TLR2-stimulating activities of 400-fold-diluted culture supernatants of 184 lactic acid bacterial strains. Each bar represents the TLR2-stimulating activity relative to that of a $100 \mathrm{ng} / \mathrm{mL}$ solution of Pam3CSK4 (100\%), a positive control, and is arranged according to activity intensity (stronger to weaker from left to right). As shown in the figure, TLR2-stimulating activity was found in MRS ferments of a variety of lactic acid bacteria. However, the activities varied widely depending on the strain.

Although the MRS medium is optimum for the growth of a variety of lactic acid bacteria, some of its components are thought to be inappropriate as ingredients for cosmetics. Therefore, we examined media made from edible and natural ingredients. Here, we used a combination of plant (e.g., asparagus) extracts and milk components (e.g., skimmed milk). The top 23 strains in Figure 1A were selected and cultured in asparagus extract containing $3 \%$ skimmed milk under static culture conditions at their preferred temperature $\left(30^{\circ} \mathrm{C}\right.$ or $37^{\circ} \mathrm{C}$ ) for $24 \mathrm{~h}$. After sterilization at $110^{\circ} \mathrm{C}$ for $20 \mathrm{~min}$, the culture supernatants were collected. The TLR2-stimulating activity of 25 -fold diluted samples was examined and is shown in Figure 1B; each bar in Figure 1B is arranged according to the activity of the supernatant, similar to Figure 1A. The figure also shows that the strains with strong activity in MRS ferments did not always have strong activity in the asparagus/milk ferments, for example, strains No. 83 and No. 62. Figure 2A shows the relationship between the TLR2-stimulating activities of MRS and asparagus/milk ferments of the top 23 strains; strain TL24 showed strong activity in both MRS and asparagus/milk ferments. As shown in Figure 2B, the dose-dependent curves for TLR2-stimulating activities in MRS and asparagus/milk ferments overlapped. Based on these data, we chose TL24 as the candidate strain for further experiments. 


\section{A}

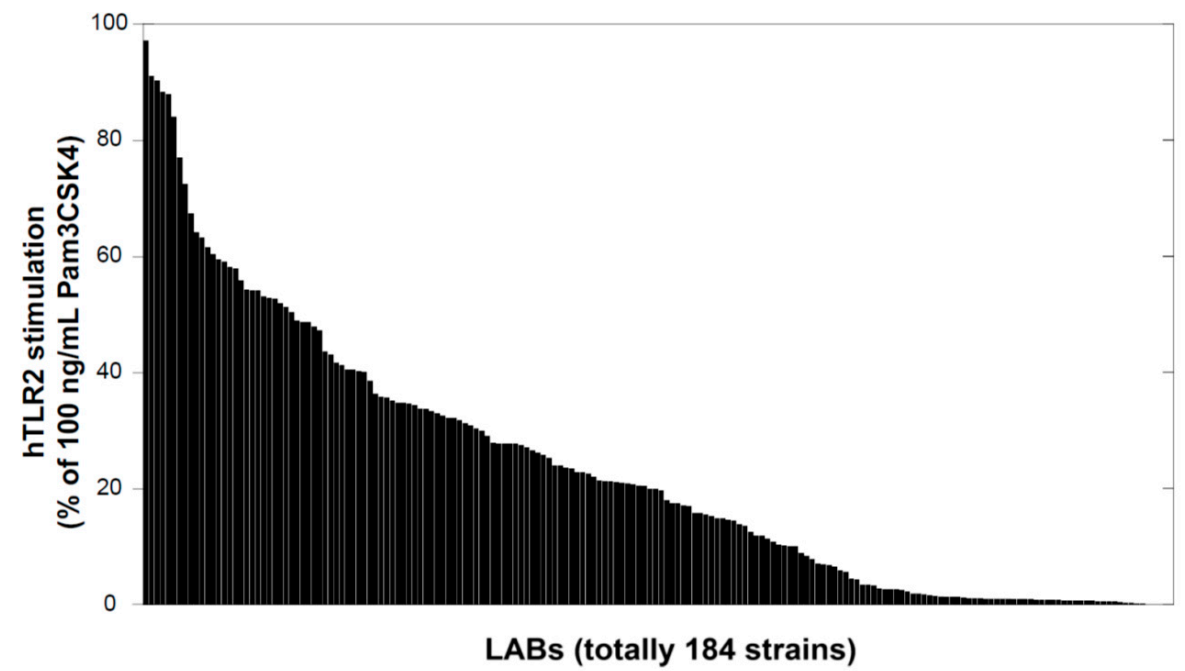

B

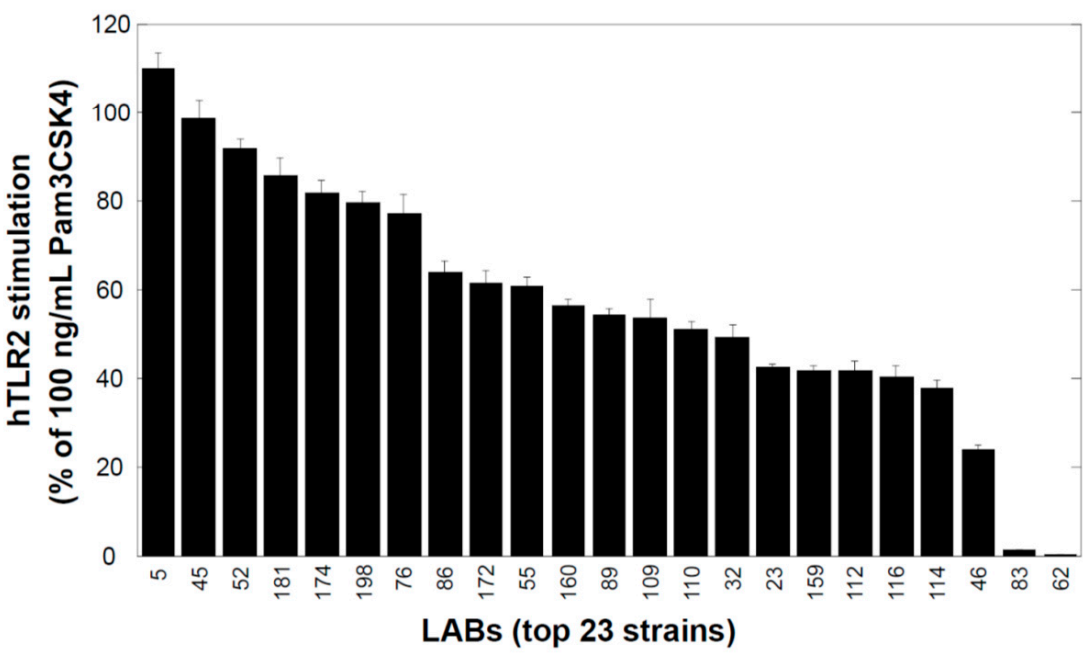

Figure 1. TLR2-stimulating activity of culture supernatants of lactic acid bacteria determined using the human TLR2 reporter cells HEK293, as described in the Materials and Methods section. (A) Effects of 400-fold dilutions of MRS ferments of 184 lactic acid bacterial strains isolated from traditional fermented foods $(n=1)$. (B) Effects of 25-fold dilutions of the ferments of the top 23 strains in Figure 1A. In this experiment, a hot water extract of asparagus (edible stem) supplemented with $3 \%$ cow skimmed milk was used as the culture medium $(n=3$; mean with S.D.). The number at the bottom of the figure is the arbitrary numbering of the strains. In both figures, each bar represents TLR2-stimulating activity relative to that of a $100 \mathrm{ng} / \mathrm{mL}$ solution of Pam3CSK4, arranged according to activity (stronger to weaker from left to right).

\subsection{Contribution of Asparagus Extract and Milk Components}

To determine the individual contributions of asparagus extract and milk components (skimmed milk and cheese whey) to the TLR2-stimulating activity of TL24 ferments, we cultured TL24 in asparagus extract, skimmed milk solution, or whey solution and compared the resulting TLR2-stimulating activities with that of cultures grown in medium consisting of both asparagus extract and milk components (skimmed milk or whey). As shown in 
Figure 3A,B, the TLR2-stimulating activity of ferments produced with single-component media was weaker than that obtained with the combined medium. Moreover, the activity of the combined-medium ferments was augmented by heat treatment (sterilization at $110{ }^{\circ} \mathrm{C}$ for $20 \mathrm{~min})$.

A

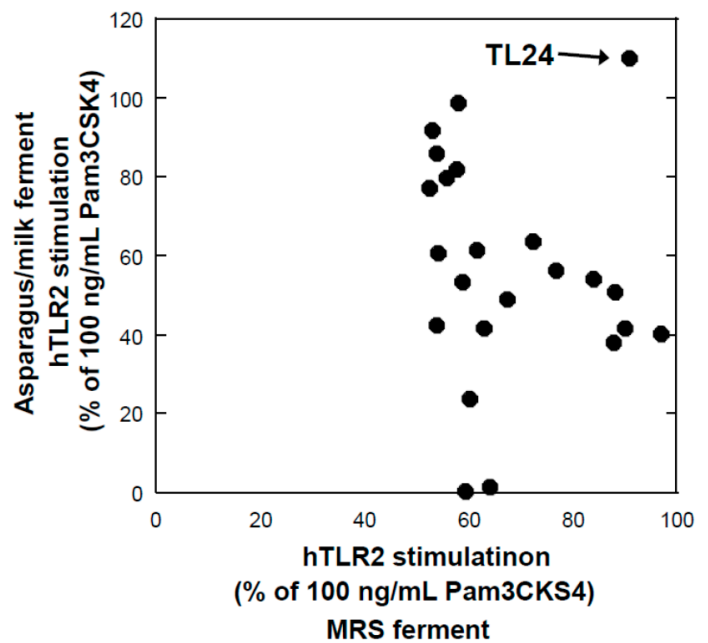

B

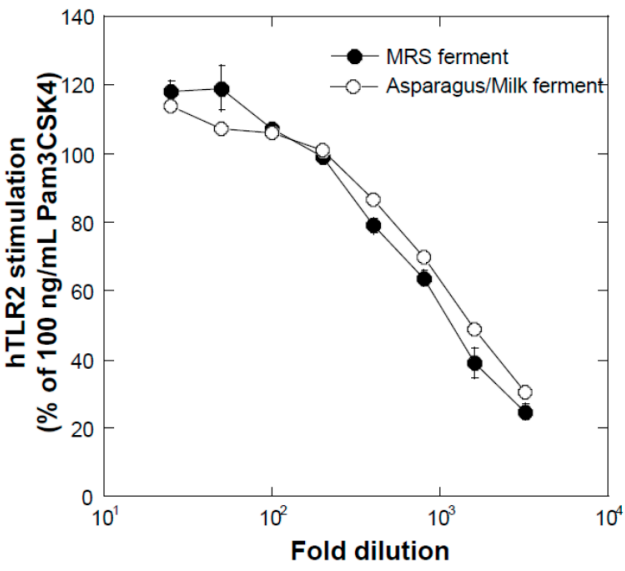

Figure 2. Relationship between two different culture media based on their TLR2-stimulating activity. (A) The TLR2-stimulating activities of the ferments obtained from two different culture media using the 23 strains described in Figure 1B were plotted based. Abscissa and ordinate represent 400fold dilutions of MRS ferments and 25-fold dilutions of asparagus/milk ferments, respectively. The strain with the highest activity in both ferments is indicated by an arrow with its name (TL24). (B) Dose-dependent curves of MRS and asparagus/milk ferments for TL24 ( $n=2$; mean with S.D.).

A

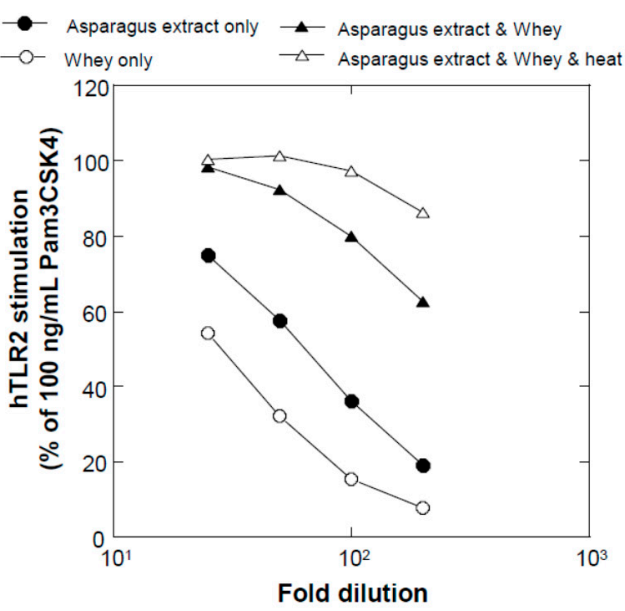

B

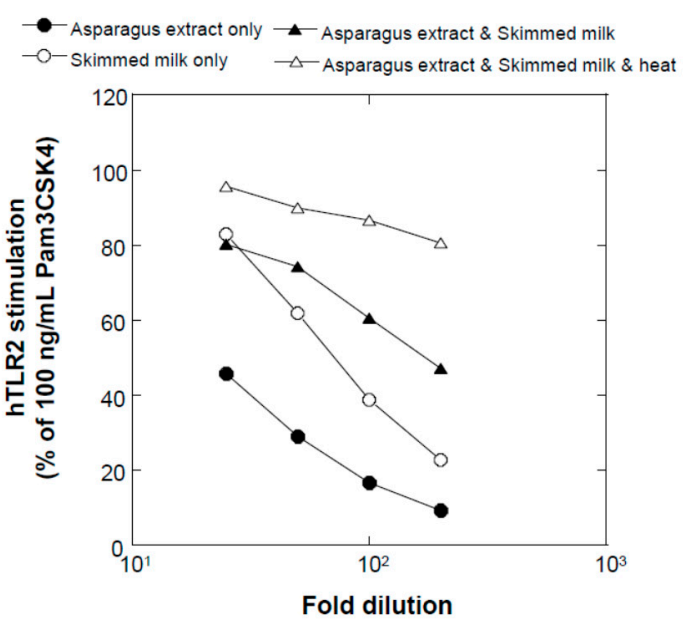

Figure 3. Contribution of asparagus extract and milk components to the TLR2-stimulating activity of TL24 ferments. (A) Hot water extract of asparagus (closed circle), 8.3\% cheese whey (open circle), or their mixture (closed triangle) was used as the medium for TL24 fermentation. After $24 \mathrm{~h}$ of static culture, the supernatants were sampled, and their TLR2-stimulating activity was examined. Before sampling, the ferments in the mixture medium were heat-treated (at $110{ }^{\circ} \mathrm{C}$ for $20 \mathrm{~min}$ ), and the activity of the supernatant was examined (open triangle). In this experiment, the media were sterilized at $75{ }^{\circ} \mathrm{C}$ for $30 \mathrm{~min}$ before fermentation. (B) We used $3 \%$ cow skimmed milk instead of cheese whey in Figure $3 \mathrm{~A}$, with sterilization at $110^{\circ} \mathrm{C}$ for $20 \mathrm{~min}$ before fermentation. 


\subsection{Comparison of Different Plant Extracts}

To evaluate the possibility of the usage of plants other than asparagus, we examined the ability of various plant extracts to support the production of a TLR2-stimulating activity. Figure 4 shows the TLR2-stimulating activity of the ferments obtained from a variety of plant extracts in combination with 3\% skimmed milk after fermentation with TL24 at $37^{\circ} \mathrm{C}$ for $24 \mathrm{~h}$ in static conditions. Almost all plant extracts, namely, extracts of asparagus (stem of edible part), broccoli (stem of edible part), cabbage (edible part), carrot (edible part, root), daikon radish (edible part, root), Chinese cabbage (edible part), apple (fruit), eggplant (fruit), cucumber (fruit), orange (fruit), tomato (fruit), and grape (fruit), could support the growth of TL24 and the production of a soluble TLR2-stimulating activity, whereas the TL24 ferments obtained using the extracts of lemon balm and cherry leaves did not show any soluble TLR2-stimulating activity. Furthermore, in almost all cases, heat treatment at $110{ }^{\circ} \mathrm{C}$ for $20 \mathrm{~min}$ augmented the TLR2-stimulating activity of the ferments.

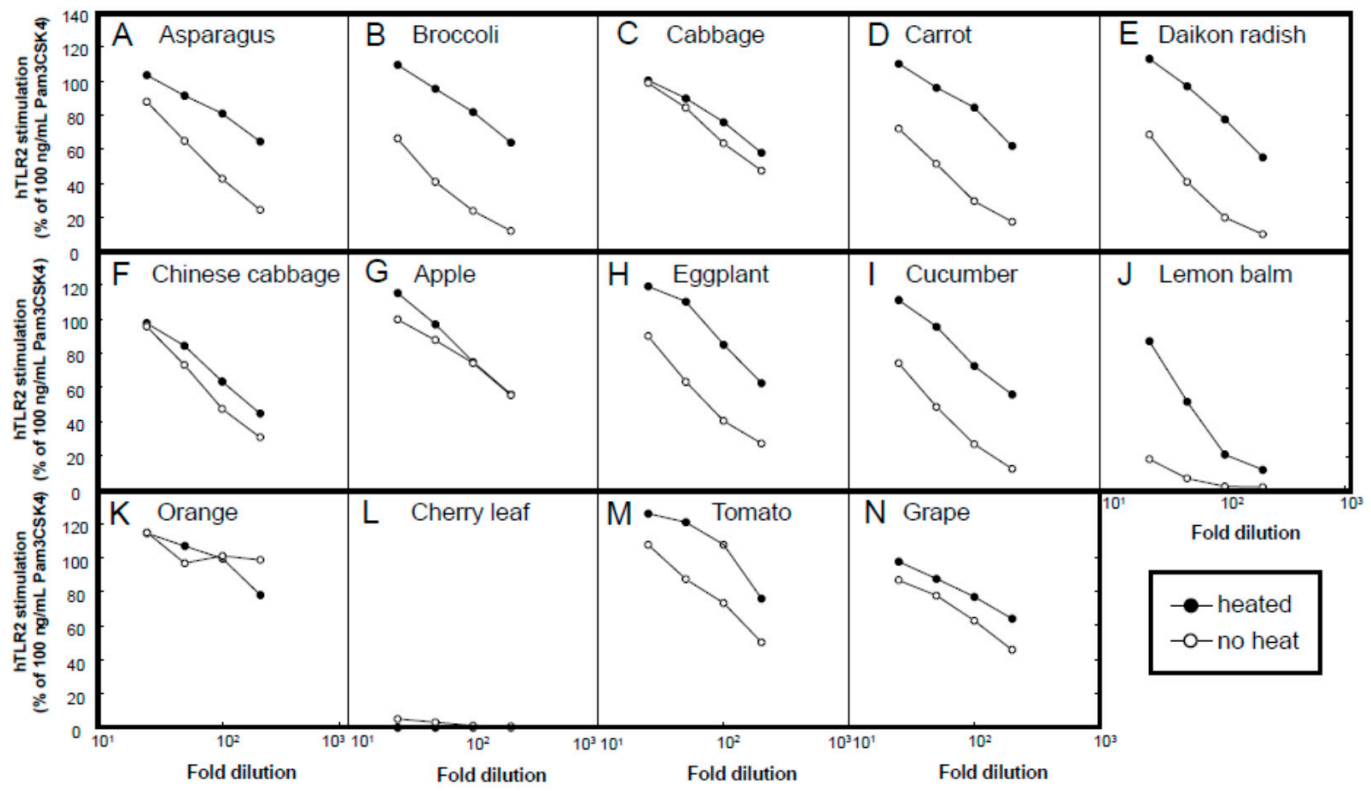

Figure 4. Comparison of different plant extracts used as the culture medium for TL24. (A) Asparagus (stem of edible part), (B) broccoli (stem of edible part), (C) cabbage (edible part), (D) carrot (edible part, root), (E) daikon radish (edible part, root), (F) Chinese cabbage (edible part), (G) apple (fruit), (H) eggplant (fruit), (I) cucumber (fruit), (J) lemon balm (leaf), (K) orange (fruit), (L) cherry leaf, (M) tomato (fruit), and (N) grape (fruit) were extracted with hot water, mixed with 3\% cow skimmed milk, and used as culture media for TL24. Open and closed circles represent the TLR2-stimulating activities of the culture supernatants before and after heat treatment at $110^{\circ} \mathrm{C}$ for $20 \mathrm{~min}$, respectively. $(\mathrm{n}=1)$.

\subsection{Comparison of Different Milk Components}

In the experiments mentioned above, cow milk was used as the source of milk components. In a subsequent experiment, we compared different milk components obtained from a variety of sources. The asparagus extract was mixed with different milk components, namely, cow skimmed milk (3\%), cow cheese whey $(8.3 \%)$, cow yogurt whey $(8.3 \%)$, cow whole milk (3\%), sheep whole milk (3\%), goat whole milk (3\%), camel whole milk $(3 \%)$, and oat milk (3\%), and was then sterilized at $75{ }^{\circ} \mathrm{C}$ for $30 \mathrm{~min}$ (whey) or at $110{ }^{\circ} \mathrm{C}$ for $20 \mathrm{~min}$ (except whey). Whey was sterilized before fermentation at $75^{\circ} \mathrm{C}$ for $30 \mathrm{~min}$, because at high temperature $\left(110^{\circ} \mathrm{C}\right)$ whey protein is denatured and forms aggregates. We used $8.3 \%(w / v)$ whey because the protein contents of 3\% skimmed milk was almost comparable to that of $8.3 \%$ cheese whey. After culturing TL24 under static conditions in the abovementioned media at $37^{\circ} \mathrm{C}$ for $24 \mathrm{~h}$, followed by sterilization at $110{ }^{\circ} \mathrm{C}$ for $20 \mathrm{~min}$, the culture supernatants were collected, and their TLR2-stimulating activities were examined. 
As shown in Figure 5, the TLR2-stimulating activities in the presence of different milk components varied. The components inducing the highest TLR-2 stimulating activity were cow skimmed milk and cow cheese whey, followed by camel whole milk. Other whole milk types, including cow milk, did not show a relevant TLR2-stimulating activity. Oat milk, a plant-derived milk-like material, induced the lowest TLR-2 stimulating activity.

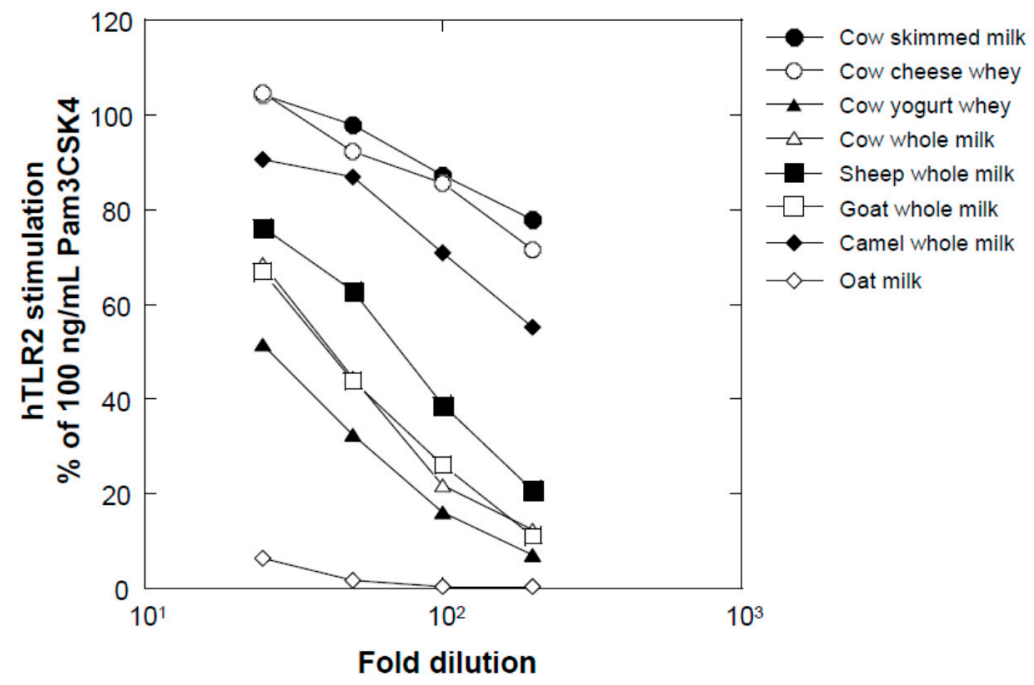

Figure 5. Comparison of different milk components used in the culture medium for TL24. Cow skimmed milk (closed circles), cow cheese whey (open circles), cow yogurt whey (closed triangles), cow whole milk (open triangles), sheep whole milk (closed squares), goat whole milk (open squares), camel whole milk (closed rhombuses), and oat milk (open rhombuses) were used as milk components mixed (individually) with a hot water extract of asparagus. The culture supernatants were sampled after TL24 fermentation at $37^{\circ} \mathrm{C}$ for $24 \mathrm{~h}$ and heat-treated at $110^{\circ} \mathrm{C}$ for $20 \mathrm{~min}$, followed by the examination of their TLR2-stimulating activities. $(n=1)$.

\subsection{Jar Fermentation}

All the experiments mentioned above were performed under static culture conditions. To evaluate the possibility of producing large amounts of ferments, taking industrial application into account, jar fermentation using a $5 \mathrm{~L}$ bioreactor was examined. Under static conditions, the acidic shift of the culture medium resulted in the aggregation of milkderived components; therefore, we used mild stirring to avoid clumping of the medium during cultivation. Figure 6A shows the TLR2-stimulating activity of TL24 ferments produced by jar fermentation, as described in the Materials and Methods section. As shown in this figure, the TLR2-stimulating activity of the jar culture ferments (closed circles) was comparable to that of the static culture ferments (Figure 2A), indicating that largescale production of ferments containing soluble TLR2-stimulating activity is feasible. In Figure 6A, the TLR2-stimulating activity of the same ferments in the presence of $1 \mu \mathrm{g} / \mathrm{mL}$ of anti-hTLR2 neutralizing antibody is shown (open circles). As clearly shown in the figure, the TLR2-stimulating activity of the ferments diminished almost completely in the presence of the neutralizing antibody, indicating that this activity was mediated by cell surface TLR2. Figure $6 \mathrm{~B}$ shows the comparison between TLR2- and TLR4-stimulating activities of the TL24 ferments produced by jar fermentation. As shown in the figure, the ferments with TLR2-stimulating activity did not stimulate TLR4, suggesting the specificity with respect to these TLRs. 
A

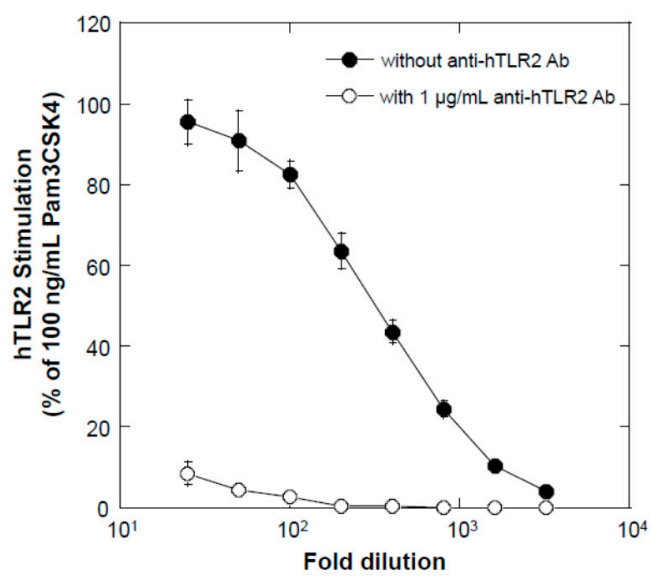

B

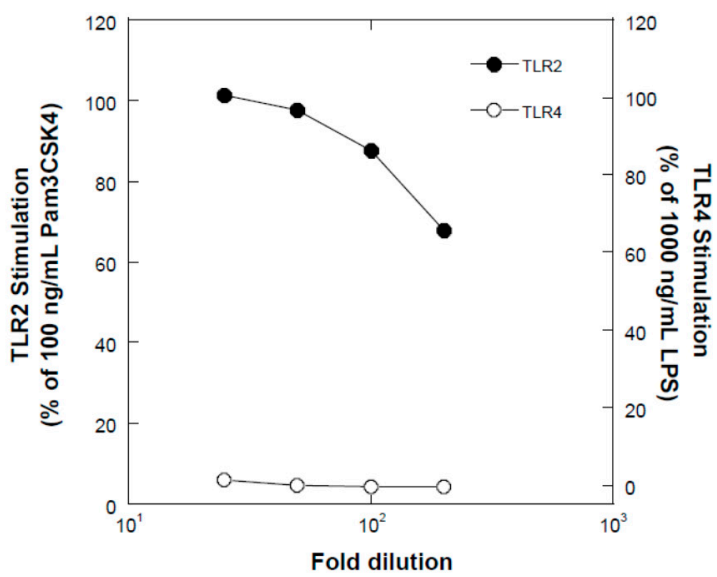

Figure 6. (A) TLR2-stimulating activity of the culture supernatant after jar fermentation. Instead of static culture conditions, jar fermentation with mild stirring (80 rpm) was employed for the fermentation of TL24 in asparagus/milk medium at $37^{\circ} \mathrm{C}$ for $24 \mathrm{~h}$. After heat treatment at $110^{\circ} \mathrm{C}$ for $20 \mathrm{~min}$, the culture supernatant was collected, and its TLR2-stimulating activity was examined (closed circles). To determine whether this activity occurred due to the stimulation of TLR2, the effect of a TLR2 neutralizing antibody was examined at a concentration of $1 \mu \mathrm{g} / \mathrm{mL}$ (open circles). ( $\mathrm{n}=3$; mean with S.D.). (B) TLR2- and TLR4-stimulating activities of the culture supernatant after jar fermentation. In addition to the TLR2-stimuating activity (closed circle), TLR4-stimulating activity was examined (open circle). $(\mathrm{n}=1)$.

\subsection{Paracellular Molecular Transfer}

Finally, we examined the functional aspects of the ferments with TLR2-stimulating activity using a paracellular molecular transfer assay [11]. NHEK (NB) cells were cultured on the membrane of a culture insert until confluency, and their differentiation was induced by $1.8 \mathrm{mM} \mathrm{CaCl}_{2}$ in the presence or absence of TL24 asparagus/milk ferments produced by jar fermentation as described above. After further incubation for $3 \mathrm{~d}$, the paracellular transfer of fluorescein was examined as described in the Materials and Methods section. As shown in Figure 7A, 2\% and 4\% of TL24 asparagus/milk ferments significantly inhibited the transfer of fluorescein through the cellular sheet of NHEK cells. To determine whether TLR2 stimulation was responsible for the inhibitory effect of TL24 asparagus/milk ferments on the paracellular transfer of fluorescein, we examined the consequences of using an anti-human TLR2 neutralizing antibody on the effect of TL24 asparagus/milk ferments. As shown in Figure 7B, $1 \mu \mathrm{g} / \mathrm{mL}$ of anti-TLR2 antibody suppressed the effect of TL24 asparagus/milk ferments, that is, its inhibition of the paracellular transfer of fluorescein. 
A

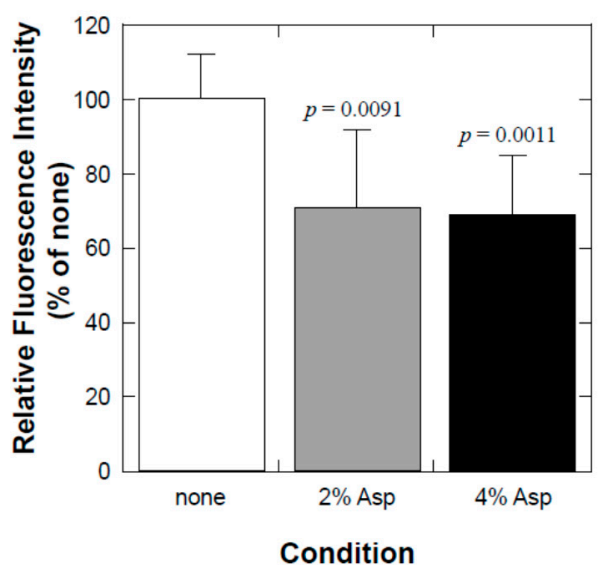

B

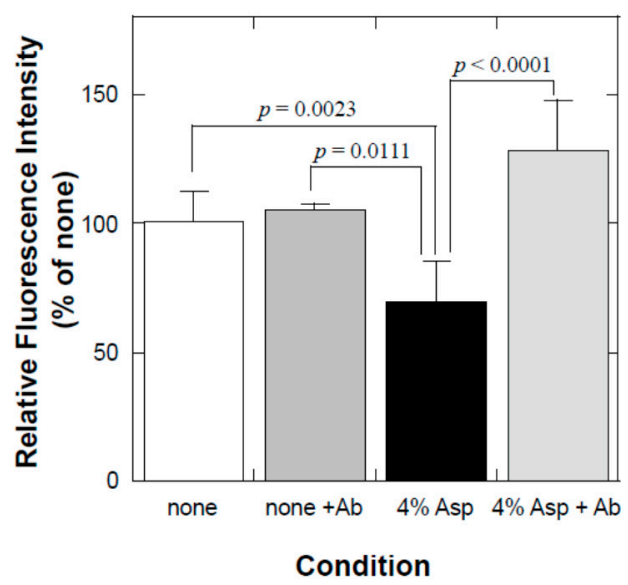

Figure 7. Effects of TL24 asparagus/milk ferments on the paracellular transfer of fluorescein through a cell sheet of human epidermal keratinocytes. (A) The effects of $2 \%$ and $4 \%$ of TL24 asparagus/milk ferments on the paracellular transfer of fluorescein were examined as described in the Materials and Methods section. Each bar represents the mean with S.D. of nine, four, and eight determinations for control (none), $2 \%$, and $4 \%$ condition, respectively. Statistical significance of the test conditions vs. control was determined using the Dunnett's test. (B) To determine whether this activity was via the stimulation of TLR2, the effect of a TLR2 neutralizing antibody was examined at a concentration of $1 \mu \mathrm{g} / \mathrm{mL}$. Each bar represents the mean with S.D. of nine, three, nine, and nine determinations for control (none), antibody only, $4 \%$ asparagus /milk ferments only, and $4 \%$ asparagus $/$ milk ferments with antibody, respectively. Statistical significance between the test groups was determined using the Scheffe's test. Significant probabilities ( $p$-values) between groups are shown in the figures, and differences between groups are considered statistically significant when the $p$-value is less than 0.05 .

\section{Discussion}

In this study, we sought cosmetically applicable novel agonists for TLR2. TLR2 is a cell surface receptor involved in innate immunity and plays an important role in recognizing microbes, including Gram-positive bacteria [12]. In keratinocytes, the main role of TLR2 is to tighten cell-cell junctions, rather than to stimulate inflammation [10,11]. Therefore, TLR2 agonists are thought to be novel cosmetic ingredients. In this study, we tried to identify appropriate lactic acid bacterial strains and their culture conditions to obtain soluble TLR2 agonists possibly suitable for cosmetics. Although the MRS medium is rich and appropriate for the culture of a variety of lactic acid bacteria, some of its components are thought to be unsuitable for the production of cosmetics. For example, the MRS medium contains "peptone," which is a digest of proteins, but in many cases, details of peptone in commercial MRS medium are not disclosed. It also contains beef extract and manganese, which are not normal ingredients in cosmetics. Furthermore, the odor of the MRS medium is not appropriate for cosmetic application, and its price is high for industrial application. Therefore, we tried to formulate a medium for lactic acid bacteria fermentation containing only compounds from natural sources, suitable for use in cosmetics.

We found that a combination of plant extracts and milk components can be used. One of the best media was the combination of a hot water extract of asparagus edible stem and milk components. Plant and milk components should not necessarily be restricted to asparagus and cow milk, respectively, because a variety of plant and milk materials could support the production of soluble TLR2 agonists. Because the activity of the ferments when asparagus extract or milk components were used individually was inferior to that obtained in their mixture, the combined usage of these materials appeared to be appropriate. Based on the results of TLR2-stimulating and paracellular molecular transfer studies, the appropriate content of the ferments in cosmetics might be $2 \%$ or higher. 
The present results suggest that the TL24 ferments containing a soluble TLR2 agonist can be used as ingredients of cosmetics. By contrast, Kim et al. [18] suggested that TLR2 might play a role in inducing inflammatory reactions in acne via stimulation by Cutibacterium acnes, the pathogenic bacterium that causes acne. However, they only demonstrated that C. acne could stimulate TLR2-transfected HEK293 reporter cells (comparable to our assay), and monocytes/macrophages were activated by C. acne via TLR2, resulting in the production of inflammatory cytokines. Based on the observation that TLR2-positive cells appeared, and their number increased in acne lesions, they speculated that TLR2 was responsible for triggering an inflammatory response in acne. However, they could not detect TLR2-positive cells in early acne lesions for up to $6 \mathrm{~h}$, though it is obvious that keratinocytes express TLR2 on their surfaces [13]. Therefore, it is reasonable to assume that C. acne does not trigger inflammation by TLR2 stimulation but augments an already existing inflammation by stimulating inflammatory cells. In fact, another commensal Gram-positive bacterium, Staphylococcus epidermidis, has been reported to be beneficial in maintaining skin health, and S. epidermidis does not trigger inflammation [19], although it is thought to stimulate TLR2. Furthermore, it has been reported that TLR2 might play a role in wound repair in $\mathrm{AD}$, rather than enhancing wounds [11]. Moreover, lactic acid bacteria, their extracts, or ferments have been widely used to promote skin health, suggesting that their intrinsic property of TLR2 stimulation is not problematic for their usage in cosmetics [20]. Collectively, these results suggest that TLR2 agonists are beneficial for non-inflamed skin, but we should be cautious about their use in the presence of inflammation. However, this is a speculation, and the safety aspects of soluble TLR2 agonists as cosmetic ingredients should be clinically evaluated in future studies.

Since TLR2 intrinsically recognizes cell surface components of Gram-positive bacteria, the ligand for TLR2 is normally thought to exist on the surface of bacteria, for example, peptide glycan, lipoteichoic acid, lipoprotein [12]. Although some fractions of cell surface ligands for TLR2 might be shed into the extracellular space, research focusing on soluble ligands for TLR2 produced by lactic acid bacteria has not yet been conducted. Regarding soluble factors produced by lactic acid bacteria to tighten cell-cell junctions, soluble proteins produced by Lactobacillus rhamnosus GG have been reported in assays involving cell sheets of the intestinal Caco2 cell line [21]. However, the molecular nature of these proteins and their underlying mechanisms for tightening cell-cell junctions have not yet been described. Based on the present study, the soluble factors produced by Lactobacillus rhamnosus GG may act as ligands for TLR2. Although, in this study, we focused on the application of soluble ligands for TLR2 to cosmetics, they might also be used as food ingredients, e.g., L. rhamnosus GG. Concerning the activation of TLRs in the gut, $\mathrm{Gu}$ et al. reported that the decrease in the barrier function of the gut induced by deoxynivalnol, a metabolite of fungi, was inhibited by TLR2 agonists [22]. On the contrary, TLR4 stimulation in the gut was reported to possibly induce the so-called leaky gut in patients with alcoholic steatohepatitis [23]. Therefore, the substances stimulating TLR2 but not TLR4 might be appropriate as food ingredients targeting the gut. The asparagus/milk ferments described in this study might have such a beneficial property.

Regarding substances responsible for the TLR2-stimulating activity of the ferments, we have not yet obtained decisive information. When we examined solvent extraction with ethyl acetate, the activity remained in the aqueous phase (data not shown), suggesting that the active components are highly hydrophilic. Synthetic lipopeptides, e.g., Pam3CSK4, have been widely used as specific agonists for TLR2, and their specificity to TLR2 was demonstrated based on X-ray crystallographic studies [24,25]. These peptides were designed based on the N-terminal structure of bacterial surface lipoprotein, and it was said that both the positively charged peptide chain and the structure of the lipid moiety were necessary [26]. Another known ligand of TLR2, lipoteichoic acid, also contains hydrophobic lipid chains and hydrophilic repeated glycerophosphate units [24]. Therefore, soluble agonists for TLR2 in the ferments might have both hydrophilic and hydrophobic structures in their molecules, although the exact structures must be elucidated in future studies. 
This study is somewhat at its early stage and with some limitations. Therefore, future studies will include (1) the elucidation of the molecular structures of soluble TLR2 agonists produced by lactic acid bacteria, including TL24, (2) obtaining morphological evidence underlying the described effect on the paracellular transfer of molecules (e.g., immunostaining of TLR2 and TJ proteins), (3) the evaluation of TLR2 agonists' effects in clinical situations, and (4) the assessment of their safety in depth, especially in a clinical setup.

Author Contributions: K.M. and Y.K. performed the experiments and wrote the manuscript. All authors have read and agreed to the published version of the manuscript.

Funding: This research received no external funding.

Data Availability Statement: The data in this article will be shared upon reasonable request to the corresponding author.

Acknowledgments: We thank Taku Miyamoto for allowing us to use his lactic acid bacteria collection as a source for screening useful strains. We also thank TechnoSuruga Laboratory Co., Ltd. (Shizuoka, Japan) for their bacterial identification efforts.

Conflicts of Interest: K.M. and Y.K. are employees of Snowden Co., Ltd.

\section{References}

1. Proksch, E.; Brandner, J.M.; Jensen, J.M. The skin: An indispensable barrier. Exp. Dermatol. 2008, 17, 1063-1072. [CrossRef] [PubMed]

2. Madison, K.C. Barrier function of the skin: "la raison d'etre" of the epidermis. J. Investig. Dermatol. 2003, 121, 231-241. [CrossRef] [PubMed]

3. Purnamawati, S.; Indrastuti, N.; Danarti, R.; Saefudin, T. The Role of Moisturizers in Addressing Various Kinds of Dermatitis: A Review. Clin. Med. Res. 2017, 15, 75-87. [CrossRef] [PubMed]

4. Yousef, H.; Alhajj, M.; Sharma, S. Skin (Integument), Epidermis. [Updated 26 July 2021]. In StatPearls; StatPearls Publishing: Treasure Island, FL, USA, 2021. Available online: https:/ / www.ncbi.nlm.nih.gov/books/NBK470464/ (accessed on 15 November 2021).

5. Brandner, J.M.; Zorn-Kruppa, M.; Yoshida, T.; Moll, I.; Beck, L.A.; De Benedetto, A. Epidermal tight junctions in health and disease. Tissue Barriers 2015, 3, e974451. [CrossRef] [PubMed]

6. Schneeberger, E.E.; Lynch, R.D. The tight junction: A multifunctional complex. Am. J. Physiol. Cell Physiol. 2004, 286, C1213-C1228. [CrossRef] [PubMed]

7. Mitic, L.L.; Van Itallie, C.M.; Anderson, J.M. Molecular physiology and pathophysiology of tight junctions I. Tight junction structure and function: Lessons from mutant animals and proteins. Am. J. Physiol. Gastrointest. Liver Physiol. 2000, 279, G250-G254. [CrossRef] [PubMed]

8. Furuse, M.; Hirase, T.; Itoh, M.; Nagafuchi, A.; Yonemura, S.; Tsukita, S.; Tsukita, S. Occludin: A novel integral membrane protein localizing at tight junctions. J. Cell Biol. 1993, 123, 1777-1788. [CrossRef]

9. Stevenson, B.R.; Siliciano, J.D.; Mooseker, M.S.; Goodenough, D.A. Identification of ZO-1: A high molecular weight polypeptide associated with the tight junction (zonula occludens) in a variety of epithelia. J. Cell Biol. 1986, 103, 755-766. [CrossRef]

10. Yuki, T.; Yoshida, H.; Akazawa, Y.; Komiya, A.; Sugiyama, Y.; Inoue, S. Activation of TLR2 enhances tight junction barrier in epidermal keratinocytes. J. Immunol. 2011, 187, 3230-3237. [CrossRef]

11. Kuo, I.H.; Carpenter-Mendini, A.; Yoshida, T.; McGirt, L.Y.; Ivanov, A.I.; Barnes, K.C.; Gallo, R.L.; Borkowski, A.W.; Yamasaki, K.; Leung, D.Y.; et al. Activation of epidermal toll-like receptor 2 enhances tight junction function: Implications for atopic dermatitis and skin barrier repair. J. Investig. Dermatol. 2013, 133, 988-998. [CrossRef]

12. Akira, S.; Uematsu, S.; Takeuchi, O. Pathogen recognition and innate immunity. Cell 2006, 124, 783-801. [CrossRef] [PubMed]

13. Kawai, K.; Shimura, H.; Minagawa, M.; Ito, A.; Tomiyama, K.; Ito, M. Expression of functional Toll-like receptor 2 on human epidermal keratinocytes. J. Dermatol. Sci 2002, 30, 185-194. [CrossRef]

14. Cario, E. Barrier-protective function of intestinal epithelial Toll-like receptor 2. Mucosal Immunol. 2008, 1 (Suppl. 1), S62-S66. [CrossRef]

15. Droemann, D.; Goldmann, T.; Branscheid, D.; Clark, R.; Dalhoff, K.; Zabel, P.; Vollmer, E. Toll-like receptor 2 is expressed by alveolar epithelial cells type II and macrophages in the human lung. Histochem. Cell Biol. 2003, 119, 103-108. [CrossRef] [PubMed]

16. Gamage, D.; Dharmadasa, R.M.; Abeysinghe, D.C.; Wijesekara, R.G.S.; Prathapasinghe, G.A.; Someya, T. Ethnopharmacological Survey on Medicinal Plants Used for Cosmetic Treatments in Traditional and Ayurveda Systems of Medicine in Sri Lanka. Evid. Based Complement. Altern. Med. 2021, 2021, 5599654. [CrossRef] [PubMed]

17. Morton, S.; Pencheon, D.; Squires, N. Sustainable Development Goals (SDGs), and their implementation: A national global framework for health, development and equity needs a systems approach at every level. Br. Med. Bull. 2017, 124, 81-90. [CrossRef] [PubMed] 
18. Kim, J.; Ochoa, M.T.; Krutzik, S.R.; Takeuchi, O.; Uematsu, S.; Legaspi, A.J.; Brightbill, H.D.; Holland, D.; Cunliffe, W.J.; Akira, S.; et al. Activation of toll-like receptor 2 in acne triggers inflammatory cytokine responses. J. Immunol. 2002, 169, 1535-1541. [CrossRef]

19. Wang, Y.; Kuo, S.; Shu, M.; Yu, J.; Huang, S.; Dai, A.; Two, A.; Gallo, R.L.; Huang, C.M. Staphylococcus epidermidis in the human skin microbiome mediates fermentation to inhibit the growth of Propionibacterium acnes: Implications of probiotics in acne vulgaris. Appl. Microbiol. Biotechnol. 2014, 98, 411-424. [CrossRef]

20. Huang, H.C.; Lee, I.J.; Huang, C.; Chang, T.M. Lactic Acid Bacteria and Lactic Acid for Skin Health and Melanogenesis Inhibition. Curr. Pharm. Biotechnol. 2020, 21, 566-577. [CrossRef]

21. Seth, A.; Yan, F.; Polk, D.B.; Rao, R.K. Probiotics ameliorate the hydrogen peroxide-induced epithelial barrier disruption by a PKC- and MAP kinase-dependent mechanism. Am. J. Physiol. Gastrointest. Liver Physiol. 2008, 294, G1060-G1069. [CrossRef]

22. Gu, M.J.; Song, S.K.; Lee, I.K.; Ko, S.; Han, S.E.; Bae, S.; Ji, S.Y.; Park, B.C.; Song, K.D.; Lee, H.K.; et al. Barrier protection via Toll-like receptor 2 signaling in porcine intestinal epithelial cells damaged by deoxynivalnol. Vet. Res. 2016, 47, 25. [CrossRef] [PubMed]

23. Li, X.; Wang, C.; Nie, J.; Lv, D.; Wang, T.; Xu, Y. Toll-like receptor 4 increases intestinal permeability through up-regulation of membrane PKC activity in alcoholic steatohepatitis. Alcohol 2013, 47, 459-465. [CrossRef] [PubMed]

24. Jin, M.S.; Lee, J.O. Structures of the toll-like receptor family and its ligand complexes. Immunity 2008, 29, 182-191. [CrossRef] [PubMed]

25. Kang, J.Y.; Nan, X.; Jin, M.S.; Youn, S.J.; Ryu, Y.H.; Mah, S.; Han, S.H.; Lee, H.; Paik, S.G.; Lee, J.O. Recognition of lipopeptide patterns by Toll-like receptor 2-Toll-like receptor 6 heterodimer. Immunity 2009, 31, 873-884. [CrossRef]

26. Offermanns, S.; Seifert, R.; Metzger, J.W.; Jung, G.; Lieberknecht, A.; Schmidt, U.; Schultz, G. Lipopeptides are effective stimulators of tyrosine phosphorylation in human myeloid cells. Biochem. J. 1992, 282 Pt 2, 551-557. [CrossRef] 